\title{
What are the risks of contracting diseases associated with chickens?1
}

Roxanne Rutledge Connelly, Christopher Mores and Amy H. Simonne ${ }^{2}$

\section{Human and Animal Diseases}

Some diseases of animals are communicable to humans. The microorganisms that cause disease can be protozoal, fungal, bacterial, chlamydial or viral (Jacob et al. 2003). Some examples of avian diseases transmissible to humans include Chlamydiosis from parakeets, pigeons, and parrots and Cryptococcosis from pigeons. Salmonella, E. coli, the viruses that cause Avian Influenza, and some encephalitis viruses are associated with chickens.

Educational embryology projects such as those offered by 4-H groups (Renner and Ouart, 2005) allow children to learn how life develops through observing eggs in an incubator. The projects require daily handling of the eggs and limited handling of the new-born chick. While nothing is risk-free, the risk of contracting Avian Influenza, Salmonella, E. coli, or mosquito-borne encephalitis by participating in embryology projects is extremely small. 
determine the presence of the disease is being conducted at two of the Department's animal health laboratories. Samples are regularly collected from backyard flocks and commercial poultry breeding flocks, and the state has a voluntary surveillance and testing program.

- Veterinarians, veterinary laboratories and those working with birds in Florida are required to report suspected cases of Avian Influenza to the Commissioner of the Florida Department of Agriculture and Consumer Services. Quarantine and other emergency plans are in place to eradicate such a disease as quickly as possible if Avian Influenza is detected.

\section{E. coli and Salmonella}

Both E. coli and Salmonella are bacteria from the Enterobacteriaceae family which are commonly found in the intestinal tracts of animals.

Salmonella are widespread in the environment and are associated with all animal species including mammals, birds, reptiles, and amphibians. Multiple outbreaks of Salmonellosis associated with handling duckling, chicks and baby chicks among children have been reported in recent years in many states (CDC, 1998; MMWR, 1997; Colorado Department of Health, 2005; Oregon State Department of Human Services, 2005), thus a risk of contracting Salmonella from chicks is always present. However, the risk can be reduced by avoiding contact with feces from these animals, carefully washing hands with soap and water after handling these animals, and avoiding hand-to-mouth contact.

Escherichia coli or E. coli is usually found in the digestive system of healthy humans and animals, and is transmitted through fecal contamination and contaminated foods and water. There are hundreds of known E. coli strains, but most of them are not disease-causing (pathogenic) strains. Illness from the pathogenic strains can range from very mild diarrhea to severe bloody diarrhea with Hemolytic Uremic Syndrome (HUS), leading to kidney failure and death. The E. coli $\mathrm{O} 157: \mathrm{H} 7$ was the source of an outbreak of several cases of E. coli O157:H7 / HUS in Florida in 2005. However, the bacteria came from animals that were part of a petting zoo exhibit at local fairs. There has not been a reported case of $E$. coli infection from chicks. Although E. coli are typically found in cattle, this organism is often used as an indicator for fecal contamination in both meat and poultry processing plants.

Some of the following prevention tips will help reduce the exposure E. coli and Salmonella or reduce the risk of infection by E. coli and Salmonella by chicks:

- The setting of very clean eggs in a sanitized incubator can reduce the potential for a problem.

- Keep the incubator away from food preparation or eating areas and sanitize the area often.

- Avoiding contact with feces from the chicks or other animals.

- Wash your hands and those of your children with soap and water immediately after touching any eggs and chicks. If soap and water are not available, use alcohol-based hand wipes and gels. Although E. coli and Salmonella can be found in young fowl, transmission from chicks to humans can be minimized by the aforementioned mean.

- Supervise children when handling eggs or baby birds. Do not allow children to nuzzle or kiss chicks, touch their mouths with their hands, or to eat or drink before washing their hands thoroughly.

If any of the children have high fever, severe diarrhea or other symptoms, contact a health care provider.

\section{Encephalitis Viruses}

Eastern equine encephalitis (EEE), St. Louis encephalitis (SLE), and West Nile (WN) viruses are present in populations of wild birds in Florida. The birds become infected through the bite of a virus-carrying mosquito. These viruses can not be transmitted from person to person or from consumption of chicken eggs or meat. There is a risk that some infected birds can transmit the virus to humans under certain strict circumstances. 
Any person living in Florida has the potential to be bitten by infected mosquitoes and a small percentage of these people can become sick from one of these viruses. The Florida Department of Health and many Florida mosquito control districts use adult chickens to monitor these viruses. These birds are considered sentinel chickens. The advantage of using chickens as sentinels for these viruses is that they are maintained in chicken coops similar to those used on farms, and if bitten by an infected mosquito, the chicken does not get sick. They form antibodies to the viruses and by testing their blood, health officials will know when there is an increase of virus in the area and can provide an early warning to humans to protect themselves from mosquito bites. However, it is important to remember that these birds are maintained outdoors in areas where they are exposed to mosquitoes. This is not the case with many educational projects such as the 4-H embryology project.

- EEE, SLE, and WN are transmitted to humans through the bite of an infected mosquito.

- For any chicken to be infected with the viruses that cause encephalitis, the chick would have to be outdoors, exposed to infected mosquitoes, and be bitten by an infected mosquito. Because chicks and eggs used for educational purposes are incubated and hatched in the classroom, the chicks are not exposed to infected mosquitoes, and therefore can NOT infect a human with any of these viruses.

- The mosquito-borne viruses that cause encephalitis are not vertically transmitted from the hen to the embryo.

- There is no evidence that newly-hatched chicks could transmit these viruses to humans.

\section{Safety Measures}

Educational projects like 4-H Embryology should include a workbook containing safety measures to ensure the health of the chicks and those handling the chicks and egg to include:

- Wash and disinfect the incubator before operating.
- Order eggs from a known, reliable source to ensure eggs and chicks are disease free.

- Ensure clean eggs are used in the projects.

- Infertile eggs and dead embryos should be removed as soon as they are observed.

- Since the primary place of exposure to Salmonella is in the home, wash hands prior to handling the eggs or birds in the classroom.

- Wash hands following direct or indirect contact with animals.

- Teach good hygiene habits to reduce the risk of E. coli and Salmonella infections.

Adherance to these simple precautions will greatly reduce the already small chance of exposure to these pathogens. Small children unable to follow these precautions should not be allowed to handle the chicks or eggs unless they are individually supervised.

\section{References}

Butcher, B. D. and R. Miles. 2003. St. Louis encephalitis - the role of chickens. EDIS Fact Sheet. http://edis.ifas.ufl.edu/VM030

Centers for Disease Control and Prevention. 2005. Key facts about Avian Influenza (Bird Flu) and Avian Influenza A (H5N1) virus. HYPERLINK "http://www.cdc.gov/flu/avian/gen-info/facts.htm" http://www.cdc.gov/flu/avian/gen-info/facts.htm

Centers for Disease Control and Prevention. 1998. Salmonella and Easter Chicks and Ducklings. http://www.cdc.gov/od/oc/media/pressrel/ salmchic.htm

Centers for Disease Control and Prevention. 2004. Salmonella Infection (salmonellosis) and Animals. http://www.cdc.gov/healthypets/diseases/ salmonellosis.htm

Colorado Department of Public Health and Environment. 2005. Multi-State Salmonella outbreaks linked to baby chicks obtained from New Mexico Hatchery. 
http://www.cdphe.state.co.us/release/2005/

042905c.html

Florida Department of Health. 2005. Florida

Department of Health E. coli 0157:H7 / HUS

Investigation Update.

http://www.doacs.state.fl.us/press/2005/

04082005.html

Holt, T. 2005. Safeguards in place to protect Florida from Avian Flu, Bronson Announces.

http://www.doacs.state.fl.us/press/2005/

02252005_2.html

Jacob, J. 1999. Viral encephalitis: the role of birds. EDIS Fact Sheet. http://edis.ifas.ufl.edu/PS045

Jacob, J. P., J. M. Gaskin, H. R. Wilson and F. B. Mather. Avian Diseases Transmissible to Humans.

EDIS Fact Sheet. http://edis.ifas.ufl.edu/PS019

MMWR. 1997. Salmonella Serotype Montevideo Infections Associated with Chicks_ Idaho, Washington, and Oregon, Spring 1995 and 1996.

46(11).

Oregon State Department of Human Services. 2005. Baby chicks identified as source of Salmonella outbreak.

http://www.oregon.gov/DHS/news/2005news/20050429a.shtml

Renner, R. L. and M. D. Ouart. 2005. 4-H Embryology Project Manual. EDIS Fact Sheet. http://edis.ifas.ufl.edu/4H030 